\title{
'Telephone Triage': a possible means of managing the after-hours patient load at primary health care facilities in South Africa
}

\author{
AA Adeniji ${ }^{a, b *}$ (D) and LH Mabuza ${ }^{c}$ \\ ${ }^{a}$ Division of Family Medicine and Primary Care, Stellenbosch University, Tygerberg, South Africa \\ ${ }^{b}$ Specialist Family Physician, Ceres Hospital, Witzenberg Substructure, Ceres, South Africa \\ ${ }^{c}$ Department of Family Medicine and Primary Health Care, Sefako Makgatho Health Sciences University, Pretoria, South Africa. \\ *Corresponding author, Email: drcostlyvictory@yahoo.com; adenijitemitope7@gmail.com

Abstract The rate at which routine patients are using the emergency department (ED) as a path to enter into the healthcare system in South Africa's community healthcare facilities and district hospitals is alarming. The increasing number of acutely presenting, less stable and routine patients are being left to the care of a reduced number of health carers after hours, at weekends and during public holidays. This circumstantial disproportionality forms the breeding ground for poor patient care, healthcare workers' burnout and inappropriate use of referral pathways.

Not all the patients occupying the ED waiting rooms actually need emergency care. A sizeable number of patients in the waiting line are routine cases that could wait without any undesirable clinical outcomes.

This opinion paper looks into the use of telephones to control after-hours patient loads at primary health care facilities in South Africa.

Keywords: community health centre, emergency department, telephone triage

\section{Introduction}

Management of the after-hours patient load at primary care emergency departments (EDs) and district hospitals is a matter for concern in South Africa.

After-hours periods and weekends are characterised by reduced numbers of healthcare workers (HCW) with an increase in the number of patients in most facilities. There were clear and predictable peaks in attendance after $16 \mathrm{~h} 00$ and at weekends in the ED of most community health centres (CHCs) in Cape Town, with a steady stream of patients presenting overnight. ${ }^{1}$

This leaves limited numbers of HCWs with an enormous number of patients. The clinicians are the most affected. Patients presenting at the EDs range from those with immediate life-threatening emergencies to those seeking basic primary care services. $^{2}$ An effective triage system is therefore imperative to sort patients by need into those who must be seen immediately and those who can safely wait. ${ }^{2}$

While 'after-hours' is designed for the care of acute emergencies at primary care facilities in South Africa, this does not, in fact, happen on the ground. This therefore challenges clinicians to search for ways and means to resolve the after-hours patient load in primary healthcare facilities in South Africa.

ED waiting rooms are more congested after hours, especially over weekends. This results from the unfinished patient load from the outpatient unit, patients who have arrived by themselves at the ED to seek health care because they felt the health centre will be less busy after hours and patients brought in by emergency medical services (EMS) after hours.

The non-emergency groups of patients combined with the actual emergency population make the management of the ED an unbearable burden for clinicians and other healthcare workers on call and on shift, respectively. This uncontrolled patient load results in an excessive workload leading to mismanagement of patients, inappropriate referrals and burnout of HCWs.

Working in the district and community health centres is a challenging task and provides a possible breeding ground for burnout and depression in medical doctors. ${ }^{3} \mathrm{CHCs}$ are uneconomical as the high, and rising, rates of ED use for non-emergency after-hours care contributes to inefficient use of resources and higher levels of spending. This is because ED visits cost the government more than non-emergency primary care visits. ${ }^{4}$

While increasing the numbers of HCWs may not be an immediately feasible option for the government, the adverse effects of this workload on patients, HCWs and the economy must not be ignored. There is an urgent need for implementation of the policy direction articulated by both provincial and national government towards prompt management of this patient load.

\section{Identifying the problems}

Health care provision is vehicled via the private and public sectors in SA; the private sector serves $16 \%$ of the population while the public sector serves $84 \% .^{5}$ This margin of disproportionality is a factor of affordability and eventually results in the over population of our 24 hour trauma centers. Healthcare provision is similarly a 24-hour service and the time at which a patient chooses to attend for treatment can only be partially influenced by departmental policies.

Doctors' working hours need to be revised to more closely resemble those of other working people. In 2011 the US Accreditation Council for Graduate Medical Education limited residents' consecutive duty to 24 hours. ${ }^{5}$ In Europe, the European Working Time Directive limits the average working week to 48 hours. $^{5}$ 
In most South African healthcare facilities, time spent in excess of 24 hours on-call is unproductive and filled with exhaustion, mismanagement and gross clinical inefficiencies. ${ }^{5}$ Accounts of doctors having accidents and dying while driving home postcall were published by Times Live magazine of June 9, 2016. ${ }^{6}$ These were reported to have been caused by fatigue resulting from an uninterrupted 30 hours on-call.

Post-call duties are actually not supposed to be mandatory after the original 24 hours on-call. Therefore, doctors should be offered the freedom to structure excess hours outside the initial 24 hours on-call to times that suit them within the same month. Such modified post-call duties could be an avenue to cater for working patients and other non-emergency pool patients with quality health care.

Worth note also is the fact that many doctors in the public sector have gradually become disinterested in on-call duties because of this rigid policy that does not take their welfare into consideration. This now leaves many of the extended hours in the public health care facilities being run by locums, who seem to show very little clinical accountability.

There is therefore a need to implement an appointment system to spread the load throughout the day and make use of the whole working day. Although many healthcare facilities now use some kind of appointment system for ambulatory patients, anybody entering the waiting rooms of many $\mathrm{CHC}$ in South Africa will at once be struck by the large number of people waiting. The effectiveness of these appointment systems is therefore in doubt as patient waiting times have not significantly reduced. Effective appointment systems have the goal of matching demand with capacity so that resources are better utilised and patient waiting times are minimised.

\section{What is telephone triage?}

There has been mounting interest in the use of telecommunication and information technology for medical purposes. ${ }^{8}$ The development of cellular mobile networks and digital telecommunication systems has edified the purpose and intent of evidence-based medicine. The transformation of medical practice is possible today because of advances in system design knowledge coupled with innovations in information technology. ${ }^{9}$ Healthcare practitioners are closer to their patients, and information sharing between clinicians is better facilitated.

Telephone triage initiatives originated mostly in the United States in response to the need to reduce escalating healthcare costs. $^{8}$ However, they became widespread in the early $1990 \mathrm{~s}$ as part of a broader strategy by many US healthcare organisations to implement demand-management strategies focused on improving consumer health knowledge and preventing the unnecessary use of expensive health resources such as EDs. ${ }^{8}$ The scope of implementation differs from one country to another because of variations in health challenges. In the United States 100 million people were estimated to have access to telephone triage in the year 2002, while in Ontario, Canada, the Ministry of Health and Long-Term Care has instituted a similar approach for a population of more than 10 million people. ${ }^{8}$

Telephone triage can therefore be seen as a patient management system utilising the telephone.
The telephone has been shown to be one of the most accessible pieces of equipment in South Africa. The Statistics South Africa 2013 survey showed that ' $81.9 \%$ of households had access to at least one cellular phone, while $12.9 \%$ of households had access to both a landline and a cellular phone. Only $0.2 \%$ of households had only a landline (Statistics SA 2013).'

According to the report, the extent of access to a telephone differed by province in South Africa: a smaller percentage of households in both the Northern Cape (13\%) and Eastern Cape (10.6\%) had access to both cellular and landline services, respectively (Figure 1).

There is a wide disparity between access to healthcare services and access to the telephone. South Africa's apartheid history leaves large racial disparities in access despite postapartheid health policy to increase the number of health facilities, including in remote rural areas. ${ }^{8}$ However, even when health services are provided free of charge, monetary and time costs of travel to a local clinic may pose a significant barrier for vulnerable segments of the population, leading to overall poorer health. ${ }^{7}$

A telephone triage system can be used in various ways for the overall benefit of patients:

- As a gatekeeping device it can regulate referral to tertiary and secondary facilities.

- Patients do not have to waste money to travel to their primary facility for issues that can be sorted out over the phone.

- Most importantly, this will be an exciting way to reduce ED patient loads between the hours of $16 \mathrm{~h} 00-08 \mathrm{~h} 00$, over weekends and on public holidays.

\section{Possible obstacles}

While it is envisaged that the introduction of telephone triage might make possible a turn-round in management of ED at primary care facilities in South Africa, the available evidence shows that it also has its own shortcomings. A systematic review on the subject revealed:

'Telephone triage may compromise patient safety, particularly if urgency is underestimated and the patient is not seen by a physician or with a delay in time. ${ }^{11}$

In the same review, 13 observational studies showed that on average telephone triage was safe in $97 \%$ (95\% Cl 96.5-97.4) of all patients accessing out-of-hours care and in $89 \%(95 \% \mathrm{Cl}$ 86.7-90.2) of patients with high urgency. ${ }^{12}$ Ten studies that used high-risk simulated patients showed that on average $46 \%$ (95\% Cl 42.7-49.8) were safe. Adverse events described in the studies included mortality ( $n=6$ studies), hospitalisation $(n=5)$, attendance at emergency department $(n=1)$ and medical errors $(n=6) .{ }^{11}$

Unfortunately, the context of most of these research articles differs greatly from the South African setting. This limits the direct transferability of these studies to the South African environment.

Introduction of telephone triage in South Africa for the control of the ED patient load will definitely open up an opportunity to identify its problems and disadvantages, which may be peculiar to South Africa. The system will need to be reviewed over time for 


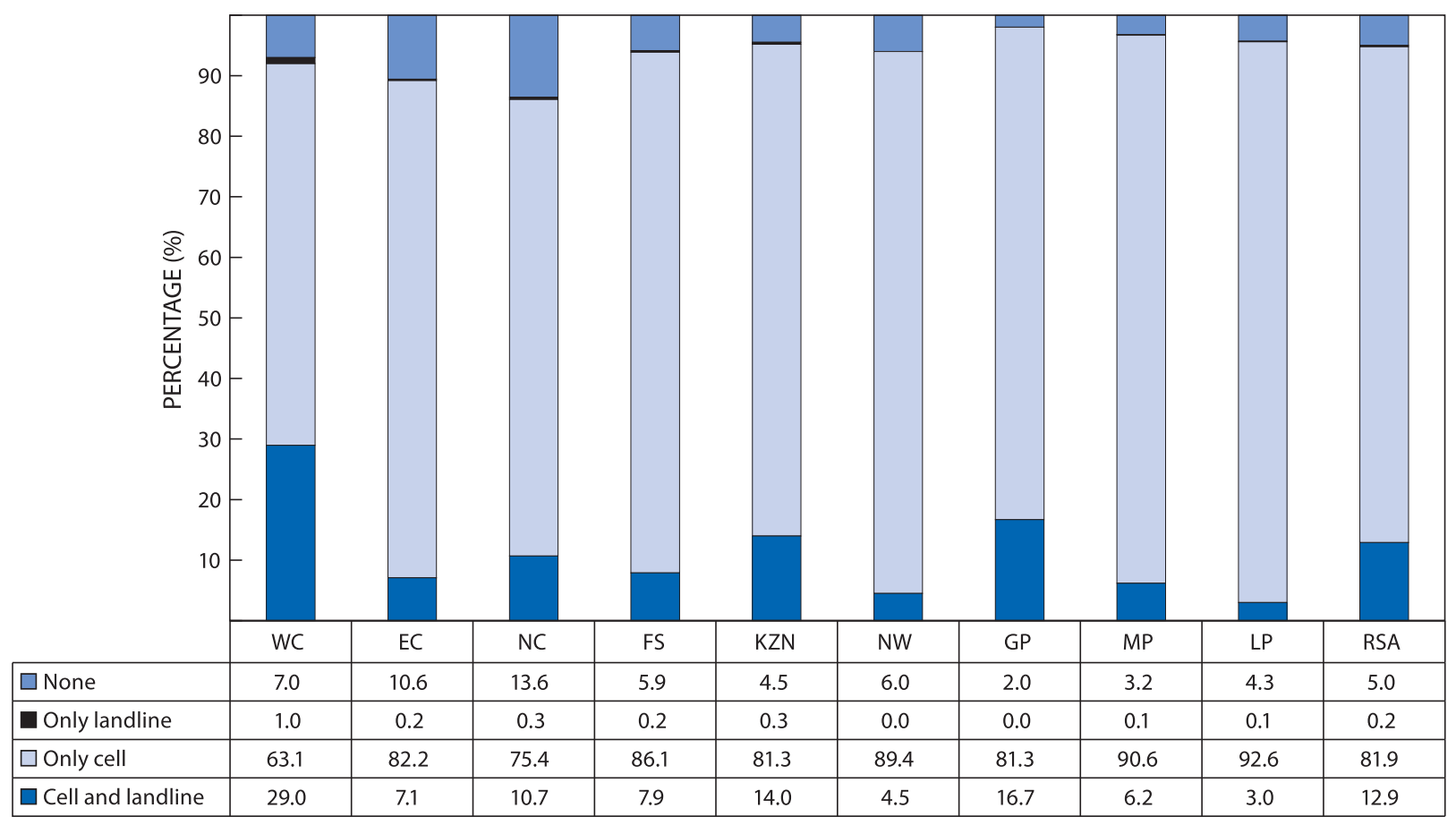

Figure 1: Percentage of households with a functional landline and cellular telephone in their home by province, 2013. Note: Statistics SA 2013: Statistical release P0318 (General household survey 2013). Page 51: Table shows percentage access to landline and cell phone as a means of communication across the nine provinces in South Africa; $95 \%$ of households have access to one or both forms of communication.

quality assurance. Telephone triage will soon be seen as a very good foundation in preparation for the phasing in of National Health Insurance (NHI) to the South African health system.

\section{Telephone triage in South Africa}

\section{Baseline situational review}

Within the Cape Town metropole 24-hour EDs operate at 10 community health centres (CHCs). These $\mathrm{CHCs}$ provide the first line of emergency care for the majority of the city's population. ${ }^{1}$ The workload, acuity and case mix of CHC EDs studied in four facilities in the Cape Town metropole showed:

- The number of patients increased at the facilities' EDs progressively from morning till midnight $24 \mathrm{~h} 00$, with the rate of increase having doubled from $16 \mathrm{~h} 00{ }^{1}$

- A sizeable number of patients are routine (given as green or yellow) and not actually urgent (orange) or emergent (red).

No research or article has been located describing other parts of SA's ED system to be less traumatic or less chaotic. The inter-rater reliability of the South African Triage System (SATS) has in fact been called into question by some researchers. South Africa's extremely high burden of HIV and tuberculosis (TB) infection, particularly in KwaZuluNatal (KZN), means that a large proportion of chronically unwell patients attend EDs. ${ }^{13}$ Their baseline physiological parameters may be grossly abnormal, e.g. patients with pulmonary TB may present with symptoms of weakness and associated immobility. ${ }^{13}$ Often, most of these types of patients occupy the 'red and orange boxes' because of their exaggerated triage early warning scores (TEWS), while the trauma/injured patient with lower TEWS due to his/her good physiological reserves, is left unattended in the 'green box'. Telephone triage may therefore serve another clinical benefit: that of modifying the priority gaps left by the questionable validity of SATS in this regard.
- Furthermore, some of the medically routine/'green' patients are often left unattended until the next morning if the ED is very busy. The 'exaggerated medical green/yellow patients' who are fortunate enough to receive a consultation are often sent home to return the following morning for their medications, X-rays or other investigations. This renders their efforts to seek help overnight an unproductive venture. Some of these patients risk being assaulted in their attempt to get to the $\mathrm{CHC}$ due to the absence of transport and the high prevalence of assault in their locations.

Telephone triage will cut unnecessary trips to the clinic, save patients' hard-earned transport fares and remove the possible security issues as a quality medical service is brought to the patients' comfort zone by means of modern technology.

\section{Implementation}

The essence of telephone triage must be that it is quality driven. The WONCA Working Party on Quality in Family Medicine defines quality as: 'The best health outcomes that are possible, given available resources, and that are consistent with patient values and preference. ${ }^{14}$ South Africa has certain resources in place that make the implementation of telephone triage a possibility. Some of these resources may, however, need to be restructured, upgraded or refashioned in order to be suitable for this purpose:

'How patients access medical care is commonly described using a triage pyramid with three different levels: Primary Care/Physician's Office/Urgent Care; Specialists/Hospitalization; and Long Term care. ${ }^{\prime 15}$

The base level of the conventional triage pyramid consists of the primary care physician's office, urgent care practices and the emergency departments. ${ }^{15}$ Usually, when care is needed, entry to the pyramid occurs at this level. An assessment of condition, 


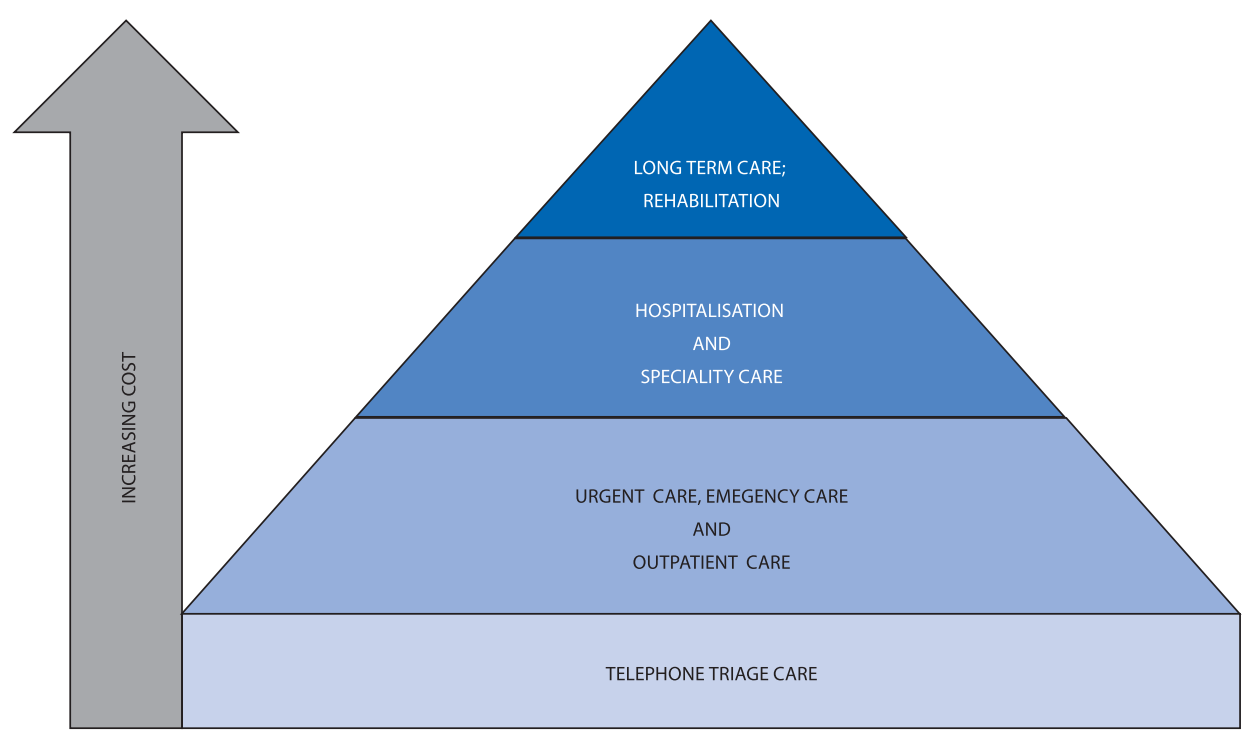

Figure 2: Conventional triage pyramid with telephone triage care as the base.

identification of treatment and prognosis for recovery are undertaken. If treatment resolves the issue the patient is released, sometimes with a follow-up appointment, and the episode is closed $^{15}$ (Figure 2).

The base of the pyramid has been coupled with a lower base occupied by the Telehealth Engagement Management System in the West. ${ }^{15}$ This fits perfectly into the level at which the integration of telephone triage is deemed feasible in the South African public health system.

Management of the lower base is vital to the actualisation of the aim of the telephone triage system. The South African Triage Scale (SATS) was developed in 2004 for pre- and in-hospital emergency units throughout South Africa. ${ }^{16}$ It was specifically designed to be used by nursing assistants and, as such, was intended to serve as a coping measure to address medical staff shortages and limited resources. ${ }^{16}$ Triage at the base of the pyramid is often carried out by triage-trained nursing personnel (professional nurse [PN], enrolled nursing assistant [ENA] or clinical nurse practitioner [CNP]) in most South African primary care emergency units and district hospitals. Such triage, however, leaves the final decision with the senior healthcare professional.

Triage at the lower base of the pyramid will require more qualified healthcare personnel to achieve a functioning telephone triage system in South Africa. Telehealth Nursing is a specialisation in First World countries that takes years to perfect. This type of nursing specialisation is not yet available in South Africa, so we are dependent on the expertise of doctors and CNPs for the final decision in telephone triage conversations. This does not mean that nurses do not have a place in telephone triage, but only that the process will have to be phased into the system in a way that will not jeopardise quality assurance.

There is no uniformity in on-call duty across the country: there are some CHCs in which two doctors are routinely on call with a family physician as a back-up or second on call. The triage in most primary care facilities is performed by an enrolled nursing assistant. $^{2}$

For telephone triage, a toll-free telephone line must be available to the triage room of all $\mathrm{CHCs}$ and district hospitals offering services on a 24-hour basis. There are several studies showing that patients who have a regular source of care use the ED more appropriately. ${ }^{17}$ The base of the pyramid will therefore be occupied by the activities of ENAs and PNs under the supervision of the medical officers on call. Routine triage can continue at this level as they can also serve as the first contact person for patients calling from home. Clinical enquiries should then be transferred to the attention of the most senior doctor on call. They can, however, attend to remedial enquiries that bear no direct impact on patients' health, e.g. patients wanting to know if they can come for completion of a form during the night.

The ENA/PN must be highly trained in communication skills. The first-contact staff must maintain professionalism at all times. The ENA should open a documentation folder (see checklist below) for the caller both to trace her/him in the existing record catalogue, and for medico-legal reasons.

Checklist:

1. Caller's name and relationship to the patient.

2. Name and folder number of the patient in the facility.

3. Location where the caller is calling from and their access to transport.

4. Chief complaint.

5. Description of signs and symptoms, onset and duration.

6. Associated symptoms.

7. Routine medications.

8. Advice given.

The most senior doctor on call may attend to the caller from point 4 (chief complaint). Calls falling into the yellow, orange or red column of the discriminator lists of SATS should essentially be attended by a medical doctor.

The authors have compiled a telephone triage table and scores (Tables 1-3), which are subject to further modification secondary to various regional differences in patients' complaints. The patients being telephone triaged could be advised to delay coming to the clinic till morning, take certain steps and delay coming to the clinic or come to the ED immediately. The choice of advice has a direct bearing on the envisaged 
Table 1: Telephone triage table

\begin{tabular}{llll}
\hline Number & Classification & Colour & Action \\
\hline 1 & $\begin{array}{l}\text { Needs } \\
\text { resuscitation }\end{array}$ & Red & Bring patient to ED immediately \\
\hline 2 & Very urgent & Orange & $\begin{array}{l}\text { Do A, B and C, then bring the } \\
\text { patient to the ED }\end{array}$ \\
\hline 3 & Urgent & Yellow & $\begin{array}{l}\text { Do A, B and C, then bring } \\
\text { patient to ED next morning }\end{array}$ \\
\hline 5 & Standard & Green & $\begin{array}{l}\text { No need for ED care, come to } \\
\text { outpatient unit next morning } \\
\text { No need for hospital care, so } \\
\text { come to outpatient unit at own } \\
\text { convenience }\end{array}$ \\
\hline
\end{tabular}

urgency of the clinical complaint. Thus the attending clinician must be competent in spot diagnosis from the history alone.

The care of a patient calling for help from the comfort of his/her home should be managed by teamwork and the welfare of these patients should be considered as a team responsibility for all staff on call.

It is important to note that the above tool has neither been tested nor validated. Its main purpose is to ensure that health information is given to callers who are in desperate need of information regarding their own ill health or that of their loved ones. The systemic parameters comprise the authors' views of the commonly presenting symptoms at EDs in South Africa. The authors consider that no bodily system can exist in isolation and that it is absolutely impossible for any sick person to score less than 2 .

Table 2: Telephone triage scores and possible action

\begin{tabular}{|c|c|c|c|}
\hline $\begin{array}{l}\text { Telephone triage } \\
\text { scores }\end{array}$ & Yes & No & If yes: Action \\
\hline Trauma & 1 & 0 & $\begin{array}{l}\text { Pressure to control } \\
\text { bleeding, splinting ....... }\end{array}$ \\
\hline Confused/unconscious & 2 & 0 & $\begin{array}{l}\text { Put in recovery position if } \\
\text { post-ictal }\end{array}$ \\
\hline Feel hot & 1 & 0 & Panadol + tepid sponge \\
\hline Breathing fast & 1 & 0 & $\begin{array}{l}\text { Expose to fresh air/MDI if } \\
\text { known asthmatic/COPD }\end{array}$ \\
\hline $\begin{array}{l}\text { Immobility (less } \\
\text { than72 hours) }\end{array}$ & 1 & 0 & $\begin{array}{l}\text { Position not to compromise } \\
\text { breathing }\end{array}$ \\
\hline Flame burns & 1 & 0 & $\begin{array}{l}\text { Cover burns with wet } \\
\text { towels/Panadol }\end{array}$ \\
\hline Hot water burns & 1 & 0 & $\begin{array}{l}\text { Cover burns with wet } \\
\text { towels/Panadol }\end{array}$ \\
\hline $\begin{array}{l}\text { Chest pain (less than } \\
2 \text { hours, not known PUD } \\
\text { patient) }\end{array}$ & 1 & 0 & $\begin{array}{l}\text { Panadol/aspirin/isordil ... } \\
\text { depending on baseline } \\
\text { medical condition }\end{array}$ \\
\hline $\begin{array}{l}\text { Vomiting ( } \times 2 \text { in last } 60 \\
\text { minutes) }\end{array}$ & 1 & 0 & ORS, gradually \\
\hline Diarrhoea & 1 & 0 & ORS, gradually \\
\hline $\begin{array}{l}\text { Irrational behaviour (first } \\
\text { episode) }\end{array}$ & 1 & 0 & Call police \\
\hline $\begin{array}{l}\text { Irrational behaviour } \\
\text { (known psychiatric } \\
\text { patient) }\end{array}$ & 1 & 0 & Call police \\
\hline Abdominal pain & 1 & 0 & Analgesics \\
\hline Bleeding & 1 & 0 & Depending on the orifice \\
\hline Total score & 15 & & \\
\hline
\end{tabular}

Note: $\mathrm{MDI}=$ Metered dose inhaler; $\mathrm{COPD}=$ chronic obstructive pulmonary disease; $\mathrm{PUD}=$ Peptic ulcer disease; ORS = Oral rehydration solution .
Table 3: Telephone triage score 15

\begin{tabular}{ll}
\hline$\square$ & Total score: 15 \\
$\square$ & Red $=6+/ 15$ \\
$\square$ & Orange $=4 \& 5 / 15$ \\
$\square$ & Yellow $=2 \& 3 / 15$ \\
$\square$ & Green $=1 / 15$ \\
$\square$ & Blue $=0 / 15$ \\
\hline
\end{tabular}

All calls during the night must be reviewed by the most senior doctor on call before the end of the duty/shift to identify which of them might need a return call and prepare for those who are expected to come for consultation in the morning. The reports on all callers, and their management, are to be submitted to the family physician for review.

\section{Conclusion}

This article has focused on providing a feasible solution to a timecritical situation in healthcare delivery in South Africa. Telephone triage is not new to family practice in First World countries. Nurses are specially trained to handle telephone triage in Canada and Australia. Recruitment is followed by several weeks of intensive training. ${ }^{8}$ Once nurses are taking calls, it takes a further three to six months to build skills to a level commensurate with the expected triage standard performance criteria. ${ }^{8}$ The objectives may differ from what is envisaged for South Africa due to the immediate critical need, but the overall outcomes may lead to an improvement in patient clinical care and the well-being of the healthcare team in terms of work overload, respectively.

Disclosure statement - No conflict of interest was reported by the authors.

Funding - This research was funded by both Stellenbosch University and Sefako Makgatho Health Sciences University.

\section{ORCID}

AA Adeniji (D) http://orcid.org/0000-0002-1339-1114

LH Mabuza (D) http://orcid.org/0000-0001-6463-4297

\section{References}

1. Lee AW, Michele T. Workload and casemix in Cape Town emergency departments. S Afr Med J. 2007 Dec;97(12):1276-80.

2. Adeniji AA, Mash B. Patients' perceptions of the triage system in a primary healthcare facility, Cape Town, South Africa. Afr J Prim Health Care Fam Med. 2016;8(1):307. doi:10.4102/phcfm.v8i1.1148

3. Rossouw L, Emsley RA, Suliman S, et al. The prevalence of burnout and depression in medical doctors working in the Cape Town Metropolitan Municipality community healthcare clinics and district hospitals of the Provincial Government of the Western Cape: a cross-sectional study. S Afr Fam Pract. 2013;55(6):567-73. doi:10.1080/20786204.2013.10874418

4. Ann SO. After-hours access to primary care practices linked with lower emergency department use and less unmet medical needs. Health Aff December 201210.1377/hlthaff.2012.0494

5. Chen-Chung $L$, Lawrence $W$. How post-call resident doctors perform, feel and areperceived in out-patient clinics. Mededuc.com. 2011 July;45(7):669-77.

6. Petersen C. Young Doctors dangerously overworked. Times Lives Magazine. http://www.iol.co.za/news/southafrica. 2034705.

7. FatmaPoniMardiah, MursyidHasanBasri. The analysis of appointment systems to reduce outpatient waiting time at Indonesia's Public Hospital. j.hrmr.20130301.06

8. Valendar FT, Peter JB, Sharon AH, et al. Telephone triage in Western Australia. MJA. 2002;176:100-3. 
9. Charles MK. Transforming care: medical practice design and information technology. Health Aff. September 2005;24(5):1296-301. doi:10.1377/hlthaff.24.5.1296

10. Statistics SA 2013: Statistical release P0318 (General household survey 2013). Page 51. Available at: https://www.statssa.gov.za/ publications/P0318/P03182013.pdf

11. Huibers $L$, Smits $M$, Renaud V, et al. Safety of telephone triage in outof-hours care: A systematic review. Scand J Prim Health Care. 2011;29 (4):198-209. doi:10.3109/02813432.2011.629150

12. McLaren Z, Ardington C, Leibbrandt M. Distance as a barrier to health care access in South Africa. Southern Africa Labour and Development Research Unit Working Paper No. 97.

13. Rosedale K, Smith ZA, Davies H, et al. The effectiveness of the South African Triage Score (SATS) in a rural emergency department. S Afr Med J. 2011;101(8):537-40.
14. Deventer C, Mash B. African primary care research: quality improvement cycles. Afr J Prim Health Care Fam Med. 2014;6(1):Art. \#598, 7pages. http://dx.doi.org/10.4102/phcfm.v6i.598

15. Lerch D. Telemedicine \& the Triage Pyramid (Infographic) MeMD blog, October 192016.

16. Dalwai MK, Twomey M, Maikere J, et al. Reliability and accuracy of the South African Triage Scale when used by nurses in the emergency department of Timergara Hospital, Pakistan. S Afr Med J. 2014 Mar 26;104(5):372-5. doi: 10.7196/samj.7604 doi:10.7196/ SAMJ.7604

17. Stein AT, Harzheim E, Costa M, Busnello E, Rodrigues LC. The relevance of continuity of care: a solution for the chaos in emergency family practice. Oxford J. 2002 April;19 (2):207-10. 